\title{
Monocular Enucleation Induces Nuclear Localization of Calcium/Calmodulin-Dependent Protein Kinase IV in Cortical Interneurons of Adult Monkey Area V1
}

\author{
Jasmin Lalonde, Pascal E. D. Lachance, and Avi Chaudhuri \\ Department of Psychology, McGill University, Montréal, Québec, Canada H3A 1B1
}

\begin{abstract}
Elevation of intracellular $\mathrm{Ca}^{2+}$ levels activates calcium/calmodulin-dependent protein kinase (CaMK) IV, which in turn plays an important role in neuroprotection and neuroplasticity. The possibility that CaMKIV is similarly involved in neocortical tissue has not been examined previously, especially with regard to the plastic nature of ocular dominance features in the primary visual cortex (area V1). We addressed this question by way of monocular enucleation (ME) to disrupt sensory input and examine CaMKIV expression changes in monkey area V1. Immunohistochemical staining of area V1 in normal infants showed a nuclear presence of CaMKIV, which did not changed after ME. However, a striking set of layer- and time-dependent changes in nuclear CaMKIV expression was observed in adult area V1 after ME. A strong increase in nuclear CaMKIV levels was evident in cortical layers II/III and VI after $1 \mathrm{~d}$ of ME and in layer IVC after $5 \mathrm{~d}$ of ME. These specific laminar changes persisted after $30 \mathrm{~d}$ of ME and, most notably, showed a columnar profile in which CaMKIV expression was linked to open-eye columns. Real-time quantitative reverse transcription-PCR and Western blot analysis showed that total amounts of CaMKIV mRNA and protein remained unchanged after ME, suggesting that a nuclear translocation may occur from the cytoplasm. Finally, double-label immunohistochemical staining with a pyramidal cell marker (SMI-32) showed that CaMKIV was absent in this subtype, whereas coincidental expression with GABA, parvalbumin, and calretinin, but not calbindin, showed its clear presence in a subset of interneurons. We propose that CaMKIV activity within diverse groups of cortical interneurons may play an important role in adaptive plastic reorganization of adult neocortical tissue.
\end{abstract}

Key words: calcium/calmodulin-dependent protein kinase IV; primary visual cortex; primate; neuroplasticity; neuroprotection; monocular deprivation; activity-dependent regulation

\section{Introduction}

Calcium/calmodulin-dependent protein kinase (CaMK) IV is an effector of molecular pathways that facilitates diverse intracellular processes, including neuroprotection and neuroplasticity (Soderling, 2000; Sée et al., 2001). Two isoforms of CaMKIV, CaMKIV $\alpha$ and CaMKIV $\beta$, were originally purified from the rat cerebellum (Ohmstede et al., 1989; Jensen et al., 1991b). The expression of CaMKIV $\beta$ is restricted to cerebellar granule cells, whereas CaMKIV $\alpha$ (referred to as CaMKIV hereon) is distributed broadly in the brain (Frangakis et al., 1991; Jensen et al., 1991b; Jones et al., 1991). Although cerebellar expression of CaMKIV is more abundant during embryonic development, hippocampal and cortical expression persists into adulthood (Jensen et al., 1991a).

CaMKIV functions in cell nuclei, in which one of its effects is to modulate transcriptional activity through cAMP response

Received Sept. 22, 2003; revised Nov. 7, 2003; accepted Nov. 7, 2003.

This work was supported by a research grant to A.C. from the Natural Sciences and Engineering Research Council of Canada (NSERC). J.L. was the recipient of scholarships from NSERC and the Québec Fonds pour la Formation de Chercheurs et l'aide à la Recherche. We thank S. Zangenehpour, C. Kogan, and R. Farivar for helpful discussions. We are also grateful to C. Milo and S. Koukoui for technical assistance.

Correspondence should be addressed to Jasmin Lalonde, Department of Psychology, McGill University, 1205 Avenue Dr. Penfield, Montréal, Québec, Canada H3A 1B1. E-mail: jasmin@ego.psych.mcgill.ca.

DOI:10.1523/JNEUROSCI.1668-03.2004

Copyright $\odot 2004$ Society for Neuroscience $\quad$ 0270-6474/04/230554-11\$15.00/0 element-binding protein (CREB) phosphorylation (Mathews et al., 1994; Sun et al., 1994). The overexpression of a dominant CaMKIV mutant in primary cultures of cerebellar neurons undergoing apoptosis has been shown to exert a neuroprotective effect (Sée et al., 2001). Conversely, overexpression of a dominant-negative form can induce apoptosis. These results have been used to put forward a model in which $\mathrm{Ca}^{2+}$ influx prevents caspase-dependent cleavage of CaMKIV, thereby promoting neuronal survival by maintaining constitutive levels of CaMKIV/CREB-dependent gene expression (Sée et al., 2001).

CaMKIV has also been shown to participate in molecular pathways that underlie neuroplastic change (Soderling, 2000). Electrophysiological recordings of cultured Purkinje neurons transfected with dominant-negative CaMKIV produces a blockade of late phase long-term depression (LTD) (Ahn et al., 1999). A similar result is obtained by inhibition of CREB but not by inhibition of cAMP-dependent protein kinase A (PKA) or mitogen-activated protein kinase pathways, suggesting that CREB activation via CaMKIV is especially necessary for late phase LTD (Ahn et al., 1999). Furthermore, CaMKIV ${ }^{-1-}$ mice show an impairment of LTD in cerebellar Purkinje neurons as well as long-term potentiation (LTP) in the hippocampus, providing in vivo support for the involvement of CaMKIV in pathways that mediate neuroplasticity (Ho et al., 2000; Kang et al., 2001). Behavioral experiments also support a role in facilitating 
neuroplasticity because CaMKIV mutant mice display significantly reduced fear memory (Wei et al., 2002) as well as consolidation/retention of hippocampus-dependent long-term memory (Kang et al., 2001).

The question of whether CaMKIV may play a comparable role in neocortical tissue has not been examined previously, especially with regard to the plastic nature of ocular dominance features in the primary visual cortex (area V1). The primate visual system serves as an excellent model for cortical neuroplasticity because of the arrangement of ocular dominance columns in area V1 (Katz and Crowley, 2002). Here, we show that CaMKIV in infant area V1 is present at high levels in the nucleus, but in normal adults, it is primarily found in the cytoplasmic compartment; that monocular visual blockade in adults produces a dynamic increase of CaMKIV in the nucleus; and that the expression of CaMKIV is mostly found in diverse subgroups of cortical interneurons. These results together suggest an activity-driven molecular engagement of neuroprotective or neuroplastic mechanisms in cortical interneurons, which may represent an ongoing process in the developing infant but one that is selectively induced by monocular blockade in the adult.

\section{Materials and Methods}

Animal preparation. Primary visual cortex (area V1) from 16 vervet monkeys (Cercopithicus aethiops) was used for this study (10 adults and 6 infants; $34-38 \mathrm{~d}$ of age). Animals were subjected to enucleation (right eye), followed by survival of 1 or $5 \mathrm{~d}$ for infants and $5 \mathrm{hr}, 1,5$, or $30 \mathrm{~d}$ for adults ( $n=2$ per time point). Two normal infant and two normal adult monkeys with binocular vision were used as controls. For enucleations, animals were sedated initially with a ketamine-xylazine mixture (7/0.6 $\mathrm{mg} / \mathrm{kg}$, i.m.) and anesthetized with sodium pentobarbital $(25 \mathrm{mg} / \mathrm{kg}$, i.v.). For harvesting of brain tissue, animals were sedated initially as above with ketamine-xylazine and killed with an overdose of sodium pentobarbital. The animals were then perfused transcardially with PBS ( $140 \mathrm{~mm} \mathrm{NaCl}, 0.27 \mathrm{~mm} \mathrm{KCl}, 1 \mathrm{~mm} \mathrm{Na} \mathrm{HPO}_{4}$, and $0.18 \mathrm{~mm} \mathrm{KH}_{2} \mathrm{PO}_{4}$ ) until completely exsanguinated. For one hemisphere, the operculum was dissected from the harvested brains, followed by separation of the overlying gray matter, and cut into small pieces. The other hemisphere was separated into three coronal blocks, with the exception of the duplicate adult monkeys in which the cortex was flattened. All brain tissue was flash frozen in a dry ice-isopentane bath and stored at $-80^{\circ} \mathrm{C}$. All experimental manipulations were performed in accordance with standard guidelines of the Canadian Council for Animal Care and were peer reviewed by an institutional Animal Care Committee.

Immunohistochemistry. Sections of area V1 from all animals were cut from the frozen blocks at a thickness of $20 \mu \mathrm{m}$ with a CM3050 cryostat (Leica, Nussloch, Germany). Frozen brain blocks from the temporal regions of some of the same animals were also sectioned to permit immunohistochemical staining of the hippocampus. The cut sections were captured on subbed glass slides, air dried, and then maintained at $-80^{\circ} \mathrm{C}$ until histological processing.

The following antisera were used for immunohistochemical staining (dilution in parentheses): (1) CaMKIV (1:500), a rabbit polyclonal antiserum raise against a recombinant protein corresponding to amino acids 328-474 mapping to the C-terminal end of human CaMKIV (Santa Cruz Biotechnology, Santa Cruz, CA); (2) NeuN (1:1000), a mouse monoclonal antiserum (Chemicon, Temecula, CA); (3) Zif268 $(1: 10,000)$, a rabbit polyclonal antiserum (a gift from R. Bravo, BristolMyers Squibb, Princeton, NJ) that has been characterized previously (Herdegen et al., 1991); (4) SMI-32 (1:5000), a mouse monoclonal antiserum (Sternberger Monoclonals, Baltimore, MD) that has been characterized previously (Sternberger and Sternberger, 1983); (5) GABA (1: 200), a rabbit polyclonal antiserum (Chemicon) that has been characterized previously (Seguela et al., 1984); (6) GABA (1:1000), a guinea pig polyclonal antiserum (Chemicon); (7) parvalbumin (1:1000), a mouse monoclonal antiserum (Swant, Bellinzona, Switzerland) that has been characterized previously (Celio et al., 1988); (8) calbindin D-28K
(1:1000), a mouse monoclonal antiserum (Swant) that has been characterized previously (Celio et al., 1990); and (9) calretinin (1:200), a goat polyclonal antiserum raise against human recombinant calretinin (Swant).

Single immunohistochemical staining was used to detect the presence of CaMKIV and Zif268 antigen. Sections were fixed for $10 \mathrm{~min}$ in $4 \%$ paraformaldehyde in $0.1 \mathrm{M}$ PBS, followed by a $5 \mathrm{~min}$ PBS wash. Slides were then incubated for $30 \mathrm{~min}$ in $3 \%$ normal sheep serum in PBS, followed by overnight incubation with mild agitation at $4{ }^{\circ} \mathrm{C}$ in primary antiserum solution (primary antiserum in $0.1 \mathrm{~m} \mathrm{PBS} / 3 \%$ normal sheep serum). Sections were then washed three times in PBS, followed by incubation for $2 \mathrm{hr}$ at room temperature in Alexa-594-conjugated anti-rabbit secondary antiserum or in biotinylated anti-rabbit secondary antiserum raised in goat (1:500 dilution in $0.1 \mathrm{~m} \mathrm{PBS} / 3 \%$ normal sheep serum). After incubation, sections were washed three times in PBS (10 min each). Sections processed with biotinylated secondary antibody were placed in a solution of avidin-biotin-conjugated HRP complex (Vector Laboratories, Burlingame, CA) for $1 \mathrm{hr}$ at room temperature. After an additional set of three washes in PBS, these sections were developed with the DAB substrate kit for peroxidase (Vector Laboratories). Finally, sections were dehydrated and coverslipped with Permount mounting medium. For fluorescence immunohistochemistry, sections were counterstained with $4^{\prime}, 6$ diamidino-2-phenylindole (DAPI) and immediately coverslipped with ProLong Anti-Fade mounting medium (Molecular Probes, Eugene, OR).

For double immunohistochemical staining, biotinylated secondary antisera were used against the SMI-32, GABA, parvalbumin, calbindin, and calretinin antisera, whereas fluorophore-conjugated secondary antisera were used against CaMKIV and NeuN antisera, unless specified otherwise. Fixed sections were washed in $0.1 \mathrm{~m} \mathrm{PBS} / 3 \%$ hydrogen peroxide for $15 \mathrm{~min}$, followed by $5 \mathrm{~min}$ in PBS, two consecutive $10 \mathrm{~min}$ incubations in $0.1 \mathrm{M}$ triethanolamine $/ 0.5 \%$ acetic anhydride, and $5 \mathrm{~min}$ in PBS. Sections were then incubated for $30 \mathrm{~min}$ in PBS $/ 3 \%$ normal serum, followed by overnight incubation with mild agitation at $4^{\circ} \mathrm{C}$ in one of the primary antibody solutions (anti-SMI-32, anti-GABA, anti-parvalbumin, anti-calbindin, or anti-calretinin in $0.1 \mathrm{M} \mathrm{PBS} / 3 \%$ normal serum). Sections were then washed three times for $10 \mathrm{~min}$ in PBS, incubated for $2 \mathrm{hr}$ at room temperature in biotinylated secondary antiserum (1:500 in $0.1 \mathrm{M} \mathrm{PBS} / 3 \%$ normal serum), and developed with the tyramide signal amplification (TSA) kit with fluorescein fluorochrome (Molecular Probes). For the NeuN antiserum, sections were incubated in Alexa- 488 secondary antiserum. Sections processed with SMI-32, GABA, parvalbumin, calbindin, and calretinin antisera were then washed three times for $5 \mathrm{~min}$ in PBS and incubated overnight with mild agitation at $4^{\circ} \mathrm{C}$ with CaMKIV primary antibody solution (anti-CaMKIV in $0.1 \mathrm{M} \mathrm{PBS} / 3 \%$ normal serum). Finally, sections were washed and incubated with secondary antiserum as described for single fluorescence immunohistochemistry.

Image processing and immunolabeling quantification. Digital images were captured with a DAGE-MTI cooled color CCD camera and Scion (Frederick, MD) series 7.0 three-chip frame grabber. Immunofluorescent images were captured with Chroma HQ Filters 41001 and 41004 for Alexa-488 and Alexa-594 fluorochromes, respectively. Adobe Photoshop 5.0 for Macintosh was used for image processing. Cell counts as well as signal intensity measures were performed with ImageJ (http://rsb.info.nih.gov/ij/).

Cell counts from the CaMKIV fluorescent images were made using two sections per experimental condition. For each section, two highresolution captures were taken, from which we obtained a total of four separate captures for each area V1 cortical layer. We placed considerable effort at ensuring that the separate captures of all cortical layers were continuous along the vertical axis. The separate Alexa-594 and DAPI captures were digitally combined to produce composite images. Equal cutoff thresholds were applied to all captures to remove background fluorescence digitally. For each capture of the CaMKIV signal, nuclear CaMKIV cell counts were performed within a $100 \mu \mathrm{m}^{2}$ grid five to six times for the Alexa-594 channel depending on the size of the layer. An overlapping signal of Alexa-594 and DAPI fluorescence was used as a criterion for nuclear localization of CaMKIV. A histogram of the Alexa594 channel was also taken for every grid count to obtain a measure of the immunostaining intensity. The measures for each capture were averaged to give the number of CaMKIV-immunopositive nuclei per $100 \mu \mathrm{m}^{2}$ 
area and the average CaMKIV immunostaining intensity. Finally, the proportional number (percentage) of CaMKIV-immunopositive nuclei per total number of cell nuclei within a 100 $\mu \mathrm{m}^{2}$ area was established by dividing the number of CaMKIV-immunopositive nuclei per the total number of nuclei displaying the DAPI signal.

Western blot analysis. Area V1 tissue was homogenized in $100 \mu \mathrm{l}$ of modified radioimmunoprecipitation assay buffer $(50 \mathrm{~mm}$ Tris- $\mathrm{HCl}$, pH 8.0, 300 mм NaCl, 0.5\% NP-40, 0.5\% deoxycholate, $1 \mathrm{~mm}$ EDTA, pH 8.0, and $0.1 \%$ SDS) supplemented with a mixture of protease inhibitors (Complete Protease Inhibitor without EDTA; Amersham) and passed through a QIA shredder (Qiagen, Hilden, Germany). The extract was supplemented with one volume of $2 \times$ Laemli buffer (100 mm Tris-HCl, $\mathrm{pH} 6.8$, $4 \%$ SDS, $0.15 \%$ bromophenol blue, $20 \%$ glycerol, and $200 \mathrm{~mm} \beta$-mercaptoethanol) and heated at $99^{\circ} \mathrm{C}$ for $4 \mathrm{~min}$. Samples were run on a $12 \%$ SDS polyacrylamide gel and blotted onto nitrocellulose (Hybond C extra; Amersham). Membranes were blocked in blotto (TBS supplemented with $0.1 \%$ Tween 20 and $2 \%$ nonfat dry milk) for $1 \mathrm{hr}$. Blots were incubated overnight with 1:3000 anti-CaMKIV or 1:1000 antiglyceraldehyde-3-dehydrogenase (GAPDH) antisera in blotto, washed extensively, and then incubated for $1 \mathrm{hr}$ with a 1:5000 dilution of anti-rabbit-HRP conjugate (Amersham) in blotto. After additional washes, the blots were incubated with chemiluminescent substrate, according the directions in the ECL kit (Amersham), and exposed to Biomax ML film (Kodak, Rochester, NY).

Real-time quantitative reverse transcription$P C R$. Total RNA from frozen area V1 tissue of monkeys at every monocular enucleation (ME) time point was isolated using the Trizol method (Invitrogen, Gaithersburg, MD). The quality of total RNA was assessed by gel capillary electrophoresis using an 2100 Bioanalyzer (Agilent). First-strand cDNA was primed with oligo $(\mathrm{dT})_{17}$, synthesized using TaqMan reverse transcription reagents (Applied Biosystems, Foster City, CA), and served as a template for real-time quantitative reverse transcriptionPCR (qRT-PCR). CaMKIV primers (forward, 5'-GGCACAGGCTGAGCTGATG-3'; reverse, 5'-CTAGTTCCAGGTCAGCCACCTTT-3') were designed using Primer Express 2.0 (Applied Biosystems) to generate an amplicon of 131 nucleotides in length. Amplicons produced during $\mathrm{qRT}$-PCR were monitored in real-time by quantification of SYBR Green I fluorescence using the ABI Prism 7000 (Applied Biosystems). The relative quantification of a transcript was performed using the comparative cycle threshold $\left(C_{\mathrm{T}}\right)$ method as described previously (User Bulletin 2, P/N 4303859B; Applied Biosystems). Briefly, the $C_{\mathrm{T}}$ of a target gene was calibrated against that of the housekeeping gene gapdh, amplified from the same sample $\left(C_{\mathrm{T}}\right.$ CaMKIV $-\mathrm{C}_{\mathrm{T}}$ $\left.\mathrm{GAPDH}=\Delta C_{\mathrm{T}}\right)$. An estimate of the relative amounts of the CaMKIV gene for each sample was then obtained by normalizing the expression against the adult normal sample $\left(\Delta C_{\mathrm{T}}\right.$ Sample $\left.-\Delta C_{\mathrm{T}}{ }^{\text {Normal }}=\Delta \Delta C_{\mathrm{T}}\right)$ and estimated using the formula fold change $=2^{-\Delta \Delta \mathrm{CT}}$.
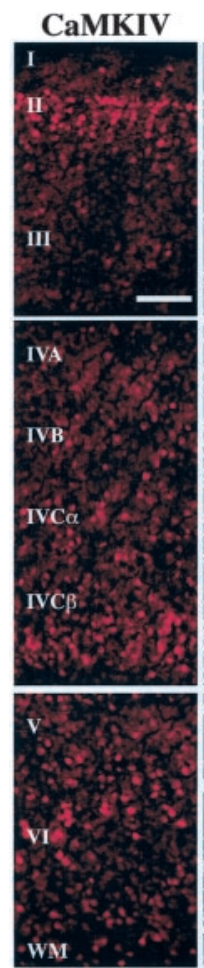

\section{A. Infant Normal}

DAPI
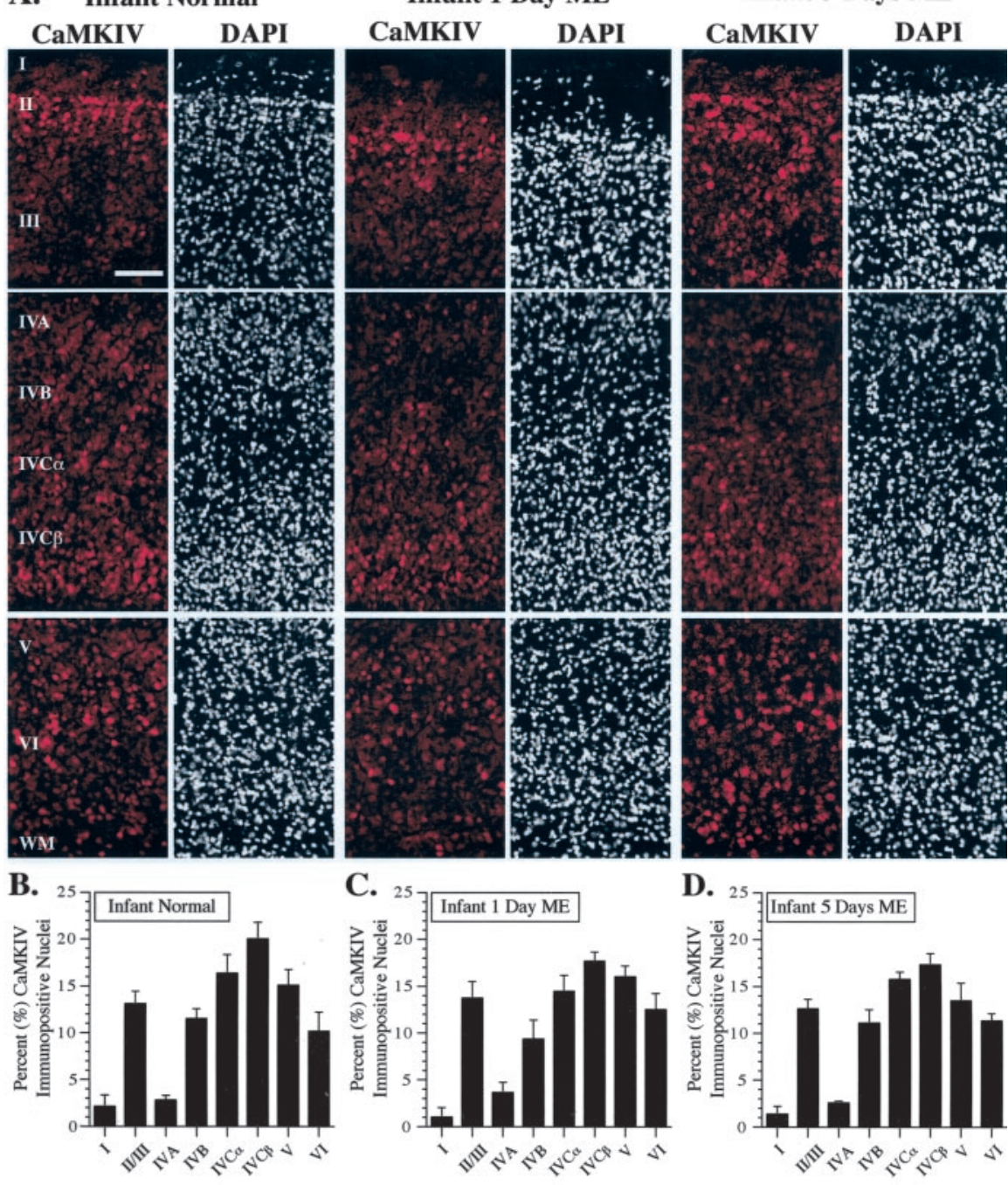

\section{E. Relative CaMKIV Immunostaining Intensity}

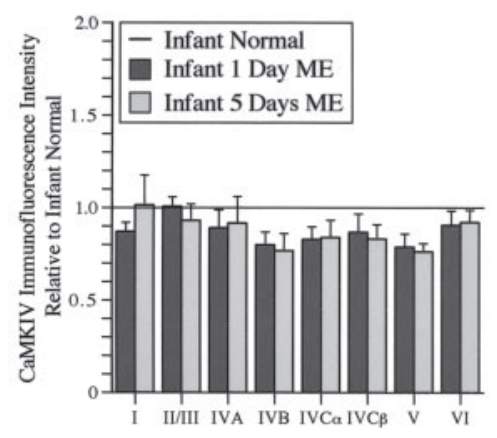

Figure 1. CaMKIV immunohistochemical staining in infant area V1 shows strong basal localization in nuclear and cytoplasmic subcompartments but is not modulated by ME. A, CaMKIV immunofluorescent staining (red) and DAPI counterstaining (white) in a normal monkey and ME infant monkeys ( 1 or $5 \mathrm{~d}$ survival). The position of each cortical layer is indicated along the left margin. WM, White matter. Scale bar, $100 \mu \mathrm{m} . B-D$, Graphical representation of the percentage of CaMKIV-immunopositive nuclei per total number of DAPI-stained nuclei for each cortical layer and ME time point in infant area V1.E, Relative CaMKIV immunostaining intensity graphed as a ratio of the corresponding layer in normal infant monkey (solid line).

\section{Results}

CaMKIV immunostaining in infant area V1

We observed CaMKIV protein expression in the primary visual cortex of three infant monkeys. In all cases, a strong immunopositive signal was observed within the nucleus, although there was also a 


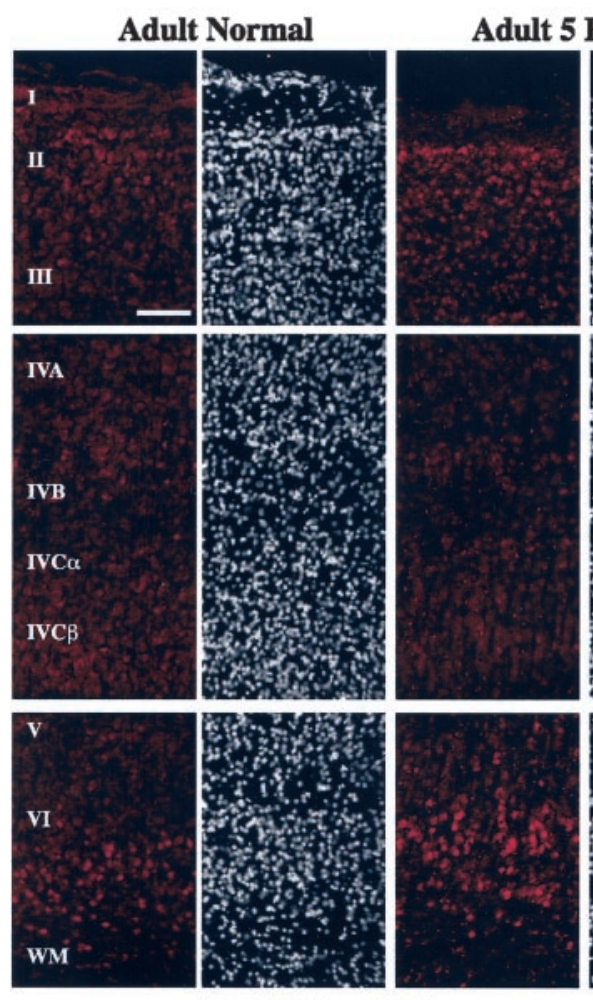

Adult 5 Days ME
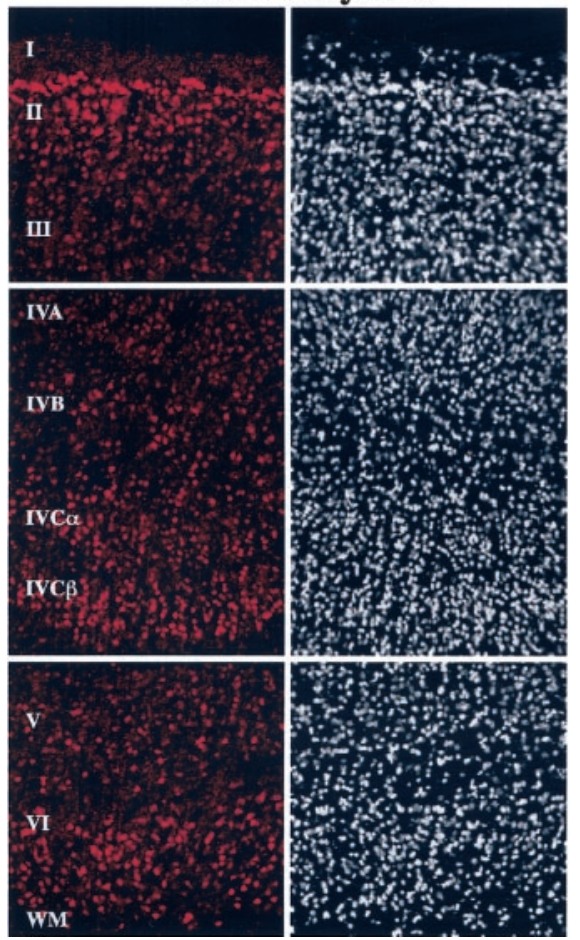

Hours ME
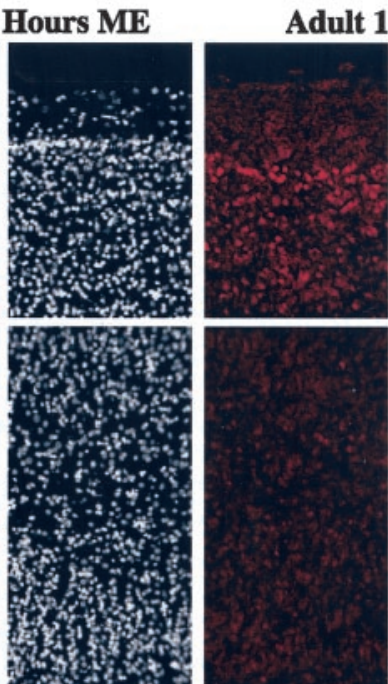

\section{Day ME}
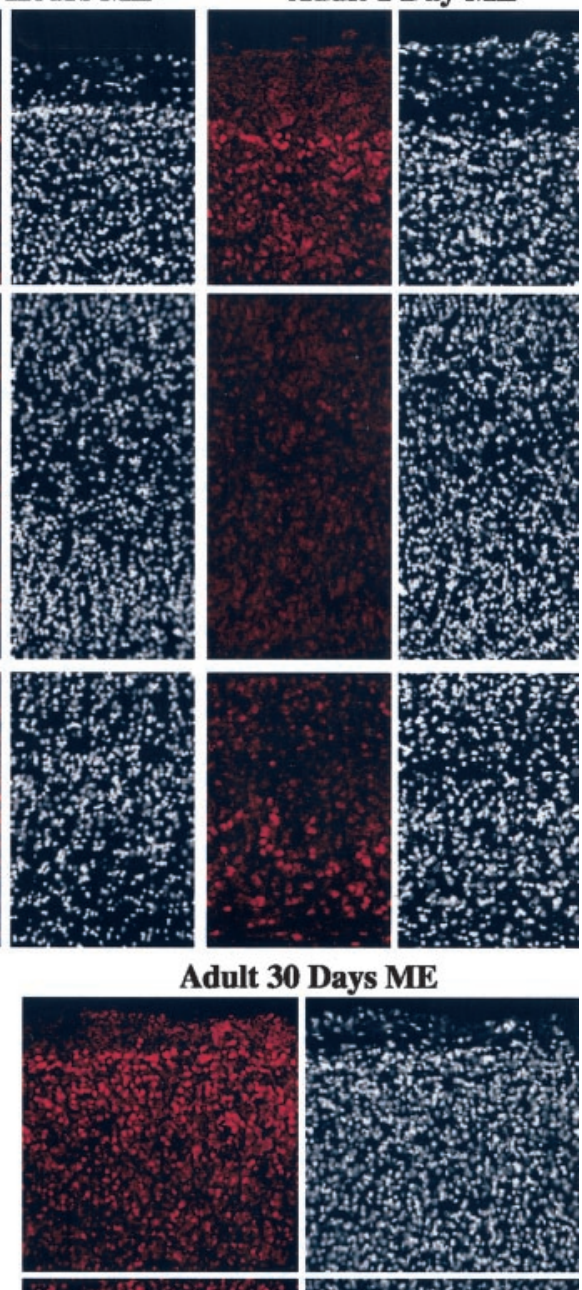

\section{Adult 30 Days ME}
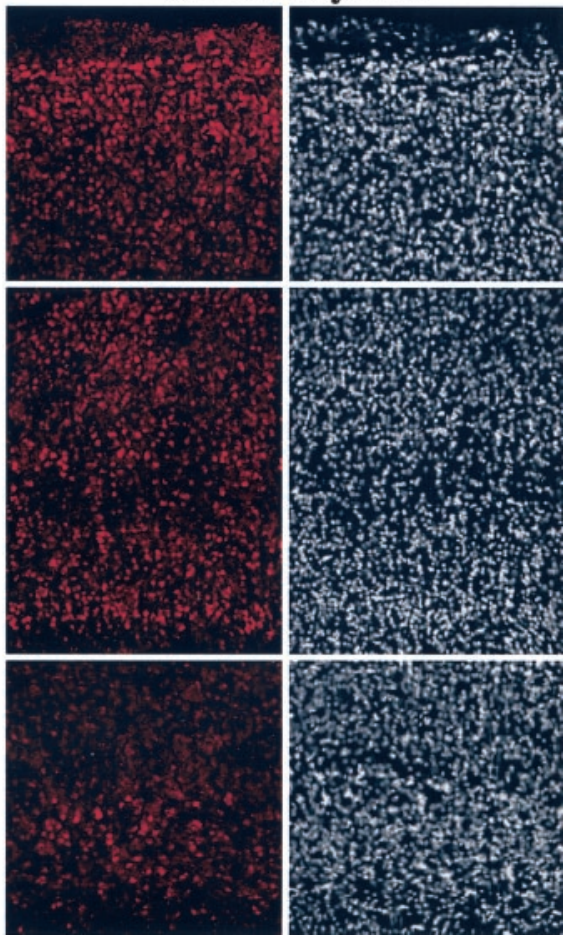

Figure 2. CaMKIV immunohistochemical staining shows that expression is localized to the cytoplasm in adult normal area V1 but that nuclear staining is dynamically increased in a temporal- and laminar-specific manner after ME. CaMKIV immunofluorescent staining (red) of adult area V1 for normal, $5 \mathrm{hr}$, and 1,5, and $30 \mathrm{~d} \mathrm{ME.} \mathrm{DAPI} \mathrm{counterstaining} \mathrm{of} \mathrm{the} \mathrm{same} \mathrm{areas} \mathrm{is} \mathrm{also} \mathrm{shown}$ (white). The position of each cortical layer is indicated by roman numerals. WM, White matter. Scale bar, $100 \mu \mathrm{m}$.

noticeable amount of staining in cytoplasmic compartments of other neurons as well. The nuclear immunostaining signals showed variations in laminar expression, with greater numbers of immunopositive neurons being evident in layers II/III, IVC, V, and VI (Fig. $1 \mathrm{~A})$. A staining gradient was observed in the supragranular layers of all three infants such that a striking number of immunopositive neurons were present at the border between layers I and II and gradually decreased toward the ventral margin of layer III.

We found that ME had no discernible impact on CaMKIV expression in area V1 of infant monkeys. Cell counts revealed that the percentage of CaMKIV-immunopositive nuclei remained stable after either 1 or $5 \mathrm{~d}$ of $\mathrm{ME}$ in comparison with the normal infant (Fig. $1 B-D$ ). Layer IVC $\beta$ displayed the highest percentage of CaMKIV-immunopositive nuclei (average between the three conditions of $18.35 \%$ of all DAPI-stained nuclei). A comparison of relative CaMKIV immunostaining intensities (Fig. $1 E$ ) showed that most cortical layers displayed somewhat weaker expression levels in both the 1 and $5 \mathrm{~d}$ ME animal (average ratio of 0.869 and 0.872 , respectively). However, this likely reflects individual or cytoplasmic staining differences because it was not corroborated by either protein-level quantitation using Western blot analyses or mRNA quantitation using qRT-PCR, as discussed later.

\section{CaMKIV immunostaining in adult} area V1

\section{CaMKIV expression in normal and short-} term ME adults

In contrast to our observations in infant area V1, nuclear CaMKIV immunostaining in the adult displayed strong dynamic changes after ME. Figure 2 shows the CaMKIV immunostaining profiles for normal and ME animals. In the normal adult, CaMKIV staining was dispersed throughout the cytoplasmic compartment. Nuclear immunostaining was barely detectable in area V1 of the normal adult, with the exception of moderate nuclear staining in layer VI. The percentage of CaMKIV-immunopositive nuclei in layer VI was $6.8 \%$ of all DAPI-stained nuclei, whereas an average of $1.71 \%$ of all DAPIstained nuclei were CaMKIV immunopositive in all of the other layers combined, except layer I, in which no significant nuclear staining was observed (Fig. 3A). At 5 hr after ME, a noticeable increase of nuclear CaMKIV-immunopositive neurons was observed in the supragranular layers II/III and a smaller increase in the infragranular layer VI (Figs. 2, 3B). A similar trend in the laminar expression profiles persisted at $1 \mathrm{~d}$ of ME, although there was now a comparable proportion of nuclear immunopositive neuron in layers II/III and VI (Figs. 2, 3C). An interesting observation at both of these ME time points was that nuclear CaMKIV expression in the internal layers (IVA-IVC and V) remained close to the low basal levels observed in the normal adult. 
Nuclear CaMKIV expression is increased after longer durations of $M E$ in adults

The immunostaining profiles in area V1 began to change considerably after longer durations of ME. At $5 \mathrm{~d}$ of ME, the number of CaMKIV-immunopositive nuclei significantly increased in not only the same layers as observed previously (layers II/III and VI) but also now in both of the subcompartments of layer IVC. This increase was clearly evident from the histological data (Fig. 2) and corroborated by the cell counts (Fig. 3D). In terms of the number of stained nuclei, nuclear immunostaining was also increased, but to a lesser extent in layers IVB and V. The most important rise in nuclear immunostaining in comparison with shorter ME durations occurred in layer IVC $\beta$, in which the percentage rose to $19.13 \%$ of all DAPI-stained nuclei, making it the highest among all layers.

CaMKIV nuclear staining continued to show similar laminar trends with more prolonged ME. The total number of immunopositive nuclei remained high across most layers after $30 \mathrm{~d}$ of $\mathrm{ME}$ (Fig. 3E). The only minor deviations in comparison with the $5 \mathrm{~d}$ ME condition appeared in layer IVA, in which a slight increase in expression was observed, and in layer VI, in which a notable decrease was apparent.

Relative intensity measures corroborated the general laminar expression trends reported above in terms of cell counts (Fig. 3F). These parallels were most apparent in those layers that showed $\mathrm{ME}$ duration-dependent changes. For example, CaMKIV expression in layers II/III and IVC increased considerably at 5 and $30 \mathrm{~d}$ of ME in comparison with the normal animal. A similar pattern was observed in the other layers as well. However, Western blot analysis suggested that total CaMKIV levels in the adult primary visual cortex of all tested animals is similar (Fig. 3G). In addition, CaMKIV mRNA levels remained unchanged in response to $\mathrm{ME}$, as discussed below. Thus, the observed changes in adult monkeys subjected to ME, especially after 5 and $30 \mathrm{~d}$, was most likely the result of nuclear concentration of the CaMKIV protein attributable to translocation from the cytoplasm.

\section{Nuclear localization of}

CaMKIV expression

Nuclear CaMKIV localization after long-term ME in adult monkeys served as the dominant factor in our immunostaining analysis. We, therefore, wished to verify more accurately the nuclear compartmentalization of CaMKIV by way of high-magnification images in area V1 after ME (Fig. 4A,B). These captures show that $5 \mathrm{~d}$ after ME, CaMKIV is primarily restricted to the nuclear compartment of a subgroup of neurons. The neuronal expression
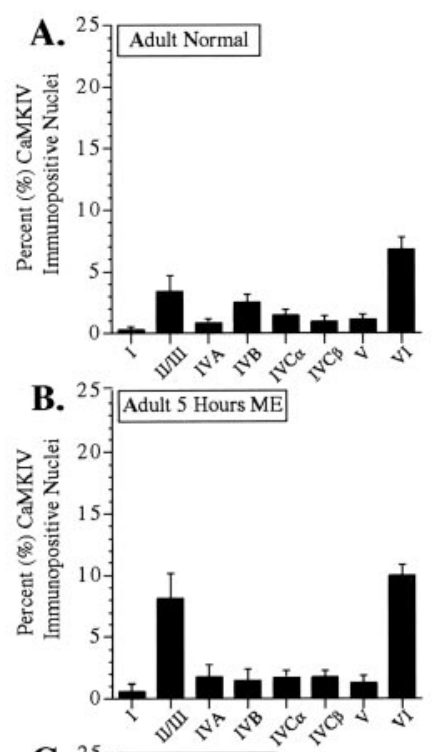

F. Relative CaMKIV Immunostaining Intensity

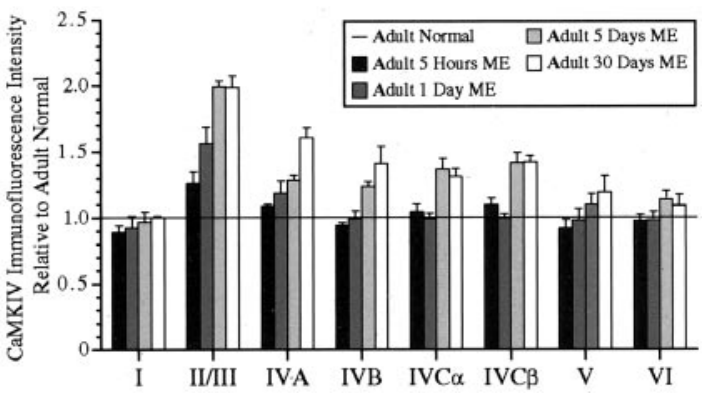

G. Western
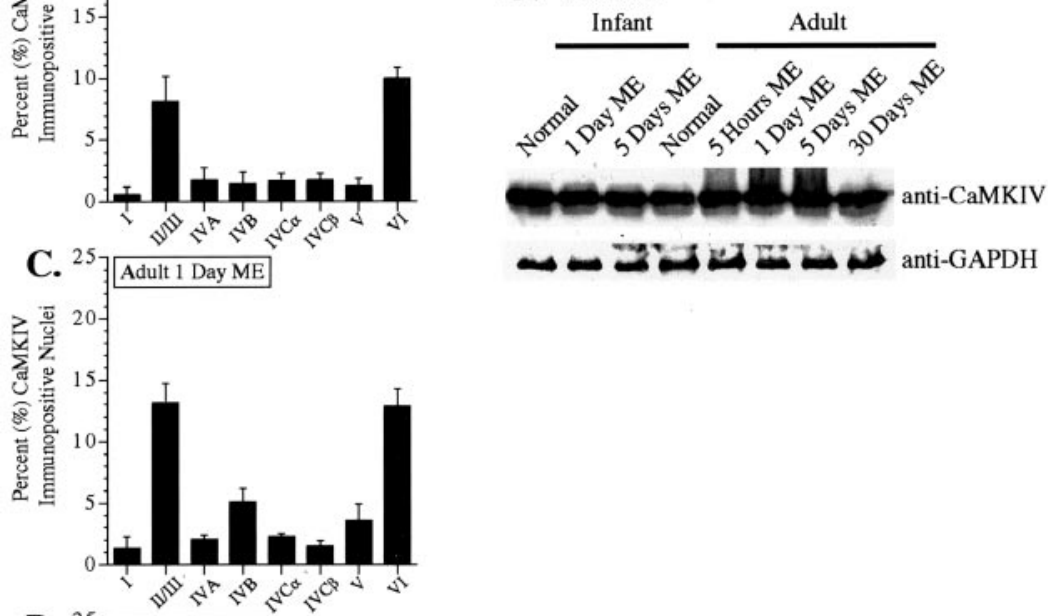

D. ${ }^{25}$ Adult 5 Days ME
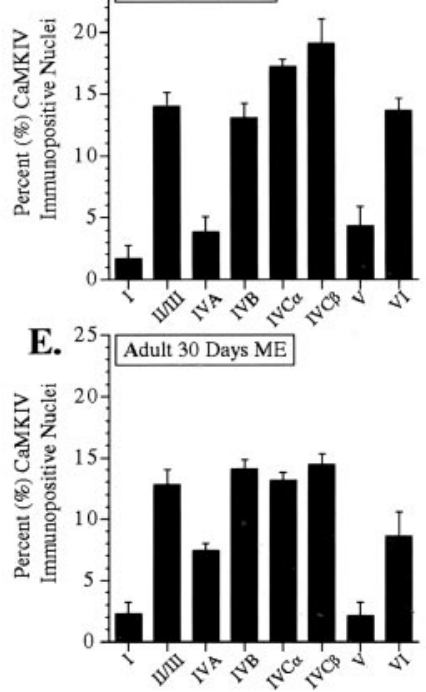

Figure 3. Counts of CaMKIV-immunopositive nuclei and relative immunostaining intensity measures in adult area V1 for all ME conditions. $A-E$, Graphical representation of the percentage of CaMKIV-immunopositive nuclei per total number of DAPI-stained nuclei for each cortical layer and ME time point in adult monkey area V1. F, Relative CaMKIV immunostaining intensity graphed as a ratio of the corresponding layer in the normal adult monkey (solid line). G, Western blot analysis of global CaMKIV protein levels in area V1 for infant and adult animals subjected to all ME conditions. GAPDH was used as loading control.

specificity was ascertained by the overlap in CaMKIV fluorescence signal with NeuN, a known nuclear protein that is found exclusively in neurons.

CaMKIV immunostaining in area V1 after long-term ME shows columnar staining

The CaMKIV staining profiles in area V1 after ME were examined closely for evidence of columnar expression. The ocular 

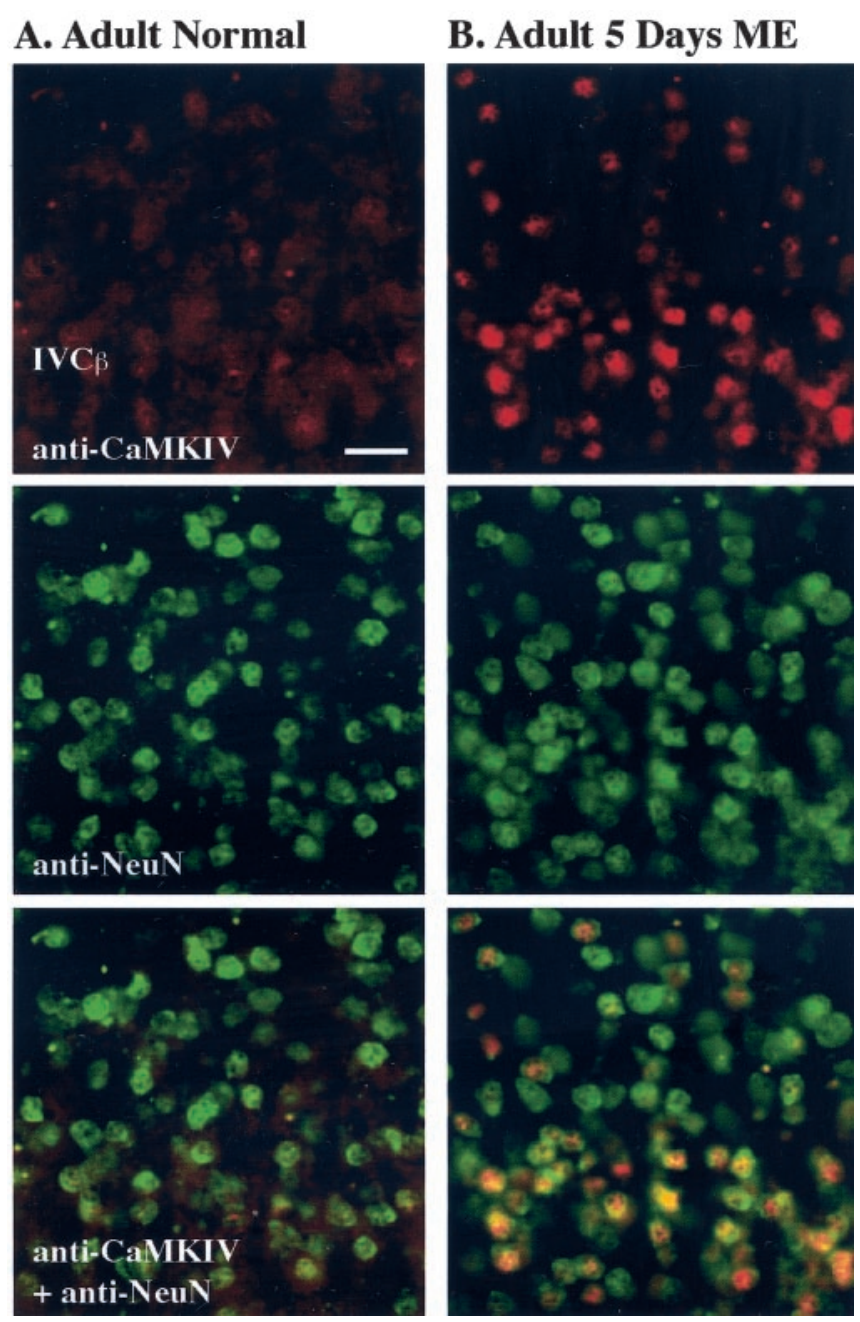

Figure 4. Immunostained sections viewed by epifluorescence microscopy show strong localization of CaMKIV in neuronal nuclei of ME adult monkeys but not in normal (non-ME) animals. Immunofluorescence signals from layer IVC $\beta$ in normal $(A)$ and $5 \mathrm{~d} M E(B)$ animals for CaMKIV (red, top panels), NeuN (green, middle panels), and colocalization (yellow, bottom panels) illustrate the specificity of the increase in nuclear (aMKIV to only a subgroup of neuronal nuclei after ME. Scale bar, $25 \mu \mathrm{m}$.

dominance (OD) architecture of area V1 provides a convenient and robust means to determine whether the expression of a particular gene product is linked to neural activity (Chaudhuri and Zangenehpour, 2002). CaMKIV immunostaining in the $30 \mathrm{~d} \mathrm{ME}$ monkeys showed columnar expression, whereas no such patterns were evident in any of the previous ME time points in both infants and adults. The columns in the $30 \mathrm{~d}$ ME animals displayed layer specificity. Although weak columnar expression was generally visible across all layers, nuclear-specific columnar staining was clearly discernible and appeared to be especially strong in layer VI. This was apparent in both coronal and flat sections of area $\mathrm{V} 1$ (Fig. $5 A, B$ ).

We next compared the CaMKIV staining patterns to adjacent sections that were immunostained for Zif268 protein to determine whether CaMKIV columns corresponded to the open or enucleated eye. It is known that Zif268 is expressed in an activitydependent manner and, therefore, selectively stains OD columns representing the intact eye (Chaudhuri et al., 1995). CaMKIV columns in area V1 were spatially coincident with Zif268 columns in both coronal and flat sections (Fig. 5). The CaMKIV columns that were visible after $30 \mathrm{~d}$ of $\mathrm{ME}$ appeared to arise from

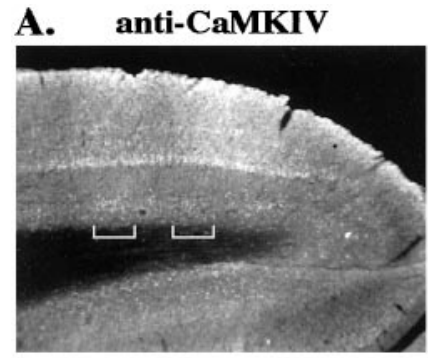

B. anti-CaMKIV

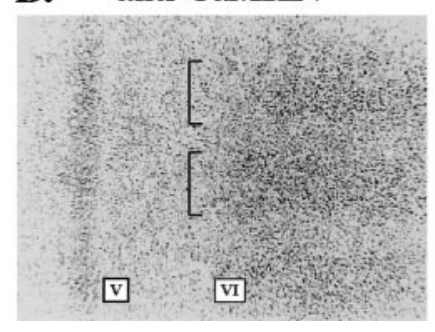

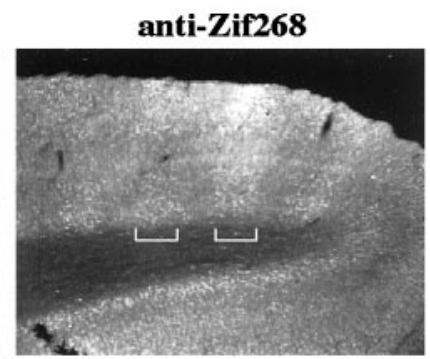

anti-Zif268

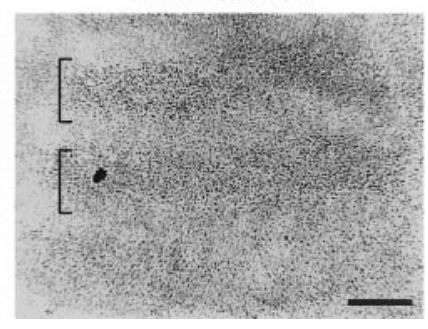

Figure 5. CaMKIV immunostaining shows a columnar expression pattern after $30 \mathrm{~d}$ of ME in adult monkeys. $A$, Fluorescent immunohistochemical staining (rhodamine fluorescence) in coronal sections of an adult $30 \mathrm{~d}$ ME monkey. Immunostaining results from two adjacent sections are shown: anti-CaMKIV (left) and anti-Zif268 (right). Note that CaMKIV-immunopositive columns are spatially coincident with those revealed by Zif268 (square brackets). B, Immunoperoxidase staining in adjacent flat sections of area $\mathrm{V} 1$ of a second $30 \mathrm{~d}$ ME monkey: CaMKIV (left) and Zif268 (right). A columnar staining pattern for CaMKIV is evident in layer VI in the flat sections and disappears at the border with layer $V$, a portion of which appears in this section. The CaMKIV columns are spatially coincident with the Zif268-immunopositive columns. Scale bar, $500 \mu \mathrm{m}$.

reduced expression levels in OD columns representing the enucleated eye because staining intensity in the intact eye columns was similar to that seen in layer VI of animals at the previous $5 \mathrm{~d}$ ME time point.

\section{CaMKIV mRNA levels in the primary visual cortex quantified} by qRT-PCR

We performed qRT-PCR analysis using two independent animal sets to quantify global CaMKIV mRNA levels in area V1 across the various experimental conditions. Expression levels of the housekeeping gene gapdh were used to normalize the qRT-PCR data. Ubiquitous genes used as standard references, including gapdh, are sometimes themselves modulated by experimental treatment (Lee et al., 2002). We, therefore, initially confirmed by Northern and Western analyses that our experimental conditions did not affect the expression of gapdh (data not shown).

CaMKIV mRNA levels in adult area V1 were seen repeatedly in the two animal sets to be (1) lower than those observed in infant primary visual cortices by a factor of at least twofold, and (2) not consistently altered in relation to ME (Table 1). Area V1 of infant monkeys also failed to show any reliable changes in CaMKIV mRNA levels in relation to ME. These results are broadly in agreement with the Western blot results for the same conditions (Fig. 3G). However, the twofold CaMKIV mRNA level difference between infant and adult V1 represented a new finding. The qRT-PCR and Western blot analyses together support the idea that the observed CaMKIV immunostaining changes seen in adult area $\mathrm{V} 1$ after ME represented a redistribution of CaMKIV from the cytoplasm to the nucleus.

\section{Morphological and cytochemical characteristics of CaMKIV} immunopositive neurons in area $\mathrm{V} 1$

Immunohistochemical staining revealed that CaMKIV was localized to neurons, the microscopic characteristics of which ap- 
Table 1. Global CaMKIV RNA expression in area V1 of infant $(\mathrm{I})$ and adult $(\mathrm{A})$ animals from all ME conditions assessed by $q R T-P C R$

\begin{tabular}{|c|c|c|c|c|c|c|c|}
\hline A norm /A norm & A $5 \mathrm{hr} / \mathrm{A}$ norm & A $1 \mathrm{~d} / \mathrm{A}$ norm & A $5 \mathrm{~d} / \mathrm{A}$ norm & A $30 \mathrm{~d} / \mathrm{A}$ norm & I norm/A norm & I1 d/A norm & I $5 \mathrm{~d} / \mathrm{A}$ norm \\
\hline \multicolumn{8}{|l|}{ Animal set 1} \\
\hline $1.00(-1.07 / 1.07)$ & $-1.15(-1.18 /-1.11)$ & $-1.39(-1.18 / 1.30)$ & $1.50(1.43 / 1.57)$ & $1.52(1.42 / 1.62)$ & $2.65(2.58 / 2.73)$ & $2.70(2.60 / 2.81)$ & $2.09(1.88 / 2.32)$ \\
\hline \multicolumn{8}{|l|}{ Animal set 2} \\
\hline $1.00(-1.04 / 1.04)$ & $1.89(1.81 / 1.96)$ & $1.42(1.36 / 1.48)$ & $1.63(1.58 / 1.68)$ & $-1.11(-1.15 / 1.08)$ & $2.11(1.99 / 2.24)$ & $3.54(3.41 / 3.66)$ & $2.81(2.65 / 2.99)$ \\
\hline
\end{tabular}

Levels of CaMKIV mRNA relative to normal adults for two independent animal sets. Corresponding error ranges are shown in parentheses. Note that the CaMKIV level was repeatedly twofold higher or more in the infant area V1 than in the normal adult. However, the CaMKIV level was not consistently affected in either direction by ME at either developmental stage. norm, Normal.

Hippocampus

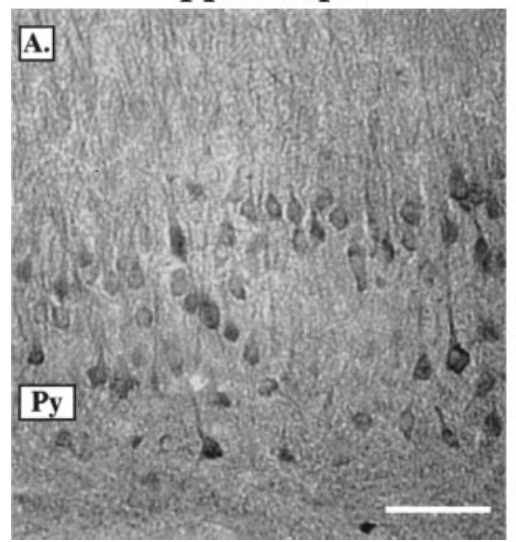

Area V1
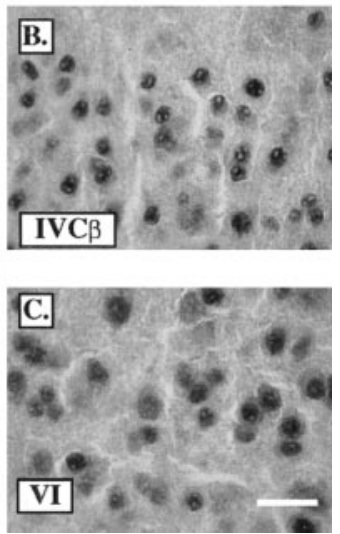

Figure 6. CaMKIV is expressed in pyramidal neurons in the hippocampus but not in area V1. A, Immunoperoxidase staining for CaMKIV in the hippocampus of an adult monkey after $5 \mathrm{~d}$ of ME. CaMKIV immunoreactivity is found in the pyramidal layer (Py) with dense CaMKIV neuropil immunostaining in layers superior to Py. Scale bar, $100 \mu \mathrm{m}$. B, C, Immunoperoxidase staining under high-magnification light microscopy is shown for layers IVC $\beta$ and VI of an adult $30 \mathrm{~d}$ ME animal. Strong nuclear CaMKIV staining is localized to small, round cells that display a different morphology than observed in the Py layer of the monkey hippocampus. Scale bar, $25 \mu \mathrm{m}$.

peared to be small and circular. This observation was mostly based on the cytoplasmic CaMKIV signal seen under fluorescent microscopy. We, therefore, suspected that CaMKIV expression was most likely confined to nonpyramidal interneurons in monkey primary visual cortex. We explored this possibility in two ways. First, we undertook comparative studies between area V1 and another brain compartment in which it is known that CaMKIV is primarily expressed in pyramidal neurons. Second, we performed colocalization staining with various cytochemical markers that are known to be selective for neuronal subtypes.

\section{Hippocampal CaMKIV immunostaining}

CaMKIV is found in the pyramidal neurons of the CA1 region of rat and mouse hippocampus (Jensen et al., 1991a; Kang et al., 2001). However, CaMKIV expression in monkey hippocampus has not been examined to date, and, therefore, we wondered whether a similar staining preference for pyramidal neurons was also present in the monkey. We performed immunohistochemical staining for CaMKIV on coronal tissue sections containing the hippocampus (Fig. 6A). A strong immunopositive signal was present within the dendrites, soma, and nuclei of CA1 hippocampal neurons. Furthermore, it is apparent that the CaMKIVimmunopositive neurons displayed pyramidal morphology, and, indeed, much of the cellular staining was confined to the pyramidal layer, with dense neuropil staining protruding into the superior layers of the CA1 region.

The results in the hippocampus suggested that if CaMKIV was indeed expressed in pyramidal neurons of area $\mathrm{V} 1$ then this should have been apparent from the morphological profiles in our stained sections. However, as observed in area V1 (Fig.
$6 B, C)$, the immunopositive neurons appeared to have nonpyramidal morphology. Therefore, to verify the cellular characteristics of CaMKIV-positive neurons, we undertook a series of colocalization experiments with endogenous markers that are known to be selective for distinct neuronal populations.

\section{CaMKIV immunostaining does not colocalize with the excitatory} cell marker SMI-32

SMI-32 is a monoclonal antibody that selectively stains nonphosphorylated neurofilament protein (Sternberger and Sternberger, 1983). It has been shown previously that SMI-32 primarily stains pyramidal neurons in monkey area $\mathrm{V} 1$ and that this feature can be used in double-labeling studies to confirm the selective expression of other neurochemical products within this neuronal subtype (Chaudhuri et al., 1995; Hof et al., 1996).

Figure 7, $A$ and $B$, shows the results of double immunofluorescence staining for CaMKIV (red fluorophore) and SMI-32 (green fluorophore) in layers IVB and VI. These area V1 layers have previously been shown to display intense SMI-32 staining (Kogan et al., 2000). Nuclear CaMKIV staining was absent in SMI-32-positive neurons. To exclude the possibility that weak cytoplasmic CaMKIV staining may have been obscured by the stronger SMI-32 signal, we undertook an additional experiment in which the CaMKIV immunocytochemical signal was amplified (TSA kit; Molecular Probes). Figure 7, $C$ and $D$, shows the SMI-32 and CaMKIV fluorescent signal captures from this step, respectively, as well as a superposition of the two signals (Fig. 7E). Again, we did not observe any instances in which excitatory neurons (e.g., pyramidal and Meynert cells) had an immunopositive CaMKIV signal in either the nucleus or cytoplasm.

\section{CaMKIV immunostaining colocalizes with GABA and other markers of interneurons in area V1}

We took the exclusion of CaMKIV in SMI-32-stained neurons as additional evidence for its confinement to nonpyramidal interneurons. To further assess this possibility, we next undertook double immunofluorescence experiments for CaMKIV and GABA colocalization (Fig. 8A,B). Anti-GABA antisera obtained from two different animals were tested. A digital superposition (right panels) of the CaMKIV (red fluorophore) and GABA (green fluorophore) signals showed that a large proportion of CaMKIV-immunopositive neurons were indeed GABAergic (yellow arrows). However, there were a number of GABAergic neurons in which CaMKIV was not detected either in the nucleus or cytoplasm, whereas another smaller group of CaMKIVimmunopositive neurons failed to clearly show GABA colocalization (white arrows). These results were broadly consistent across signals obtained with both of GABA antisera.

This result led us to perform additional verification to assess more accurately the dynamic expression of CaMKIV in different groups of GABAergic interneurons. We used antiserum against parvalbumin, calbindin, and calretinin, three calcium-binding proteins that are well known to be specific to distinctive populations of GABAergic interneurons in the adult neocortex of mon- 

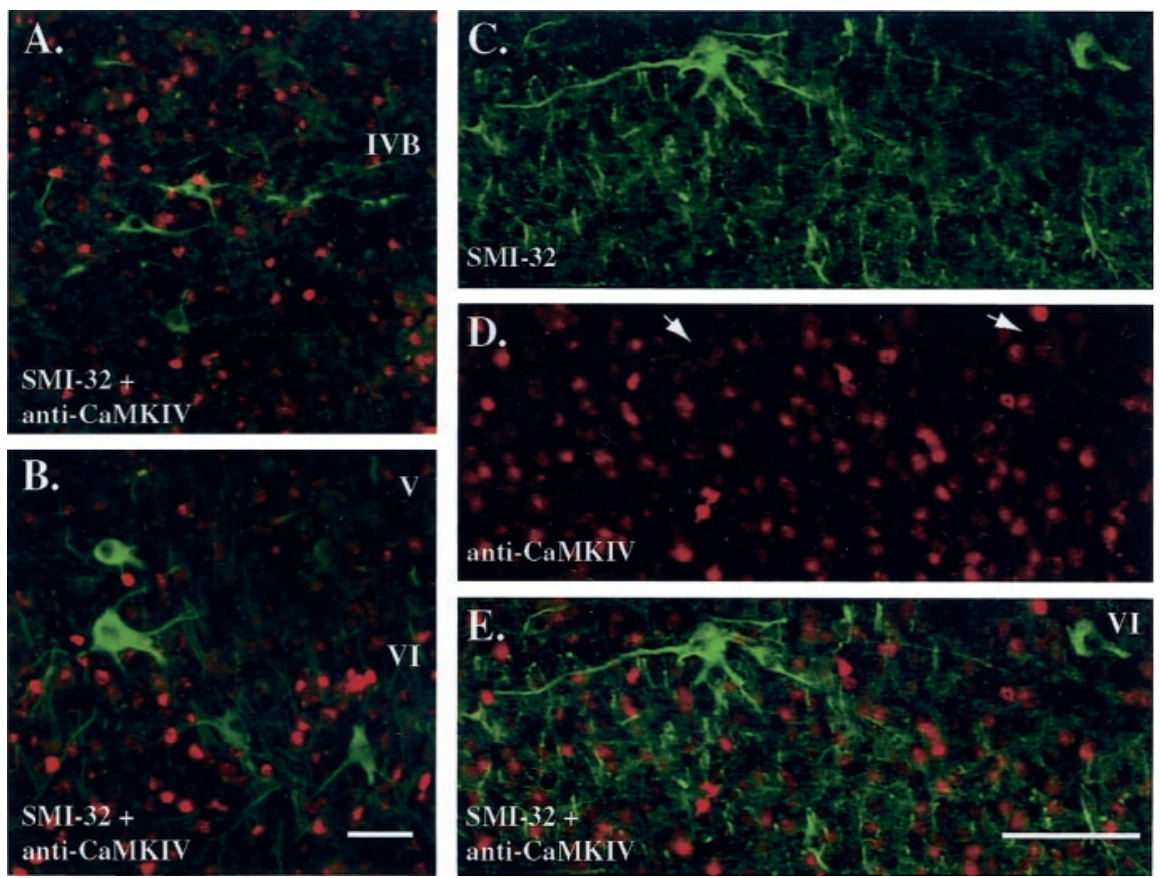

Figure 7. CaMKIV immunofluorescence signals do not colocalize with those of SMI-32, a maker of pyramidal neurons. $A, B$, Double immunofluorescence staining of SMI-32 (green) and a nonamplified CaMKIV signal (red) in layers IVB and VI of area V1 in a $5 \mathrm{~d}$ ME adult monkey. Scale bar, $50 \mu \mathrm{m}$. C, D, Immunofluorescence staining for SMI-32 (C, green), an amplified CaMKIV signal using the TSA method ( $D$, red) in a second $5 \mathrm{~d}$ ME adult monkey. $E$, Digitally superimposed image of CaMKIV and SMI-32 staining showing a lack of spatial coincidence in the two signals. White arrows in D indicate the absence of CaMKIV immunostaining in SMI-32-positive neurons. Scale bar, $100 \mu \mathrm{m}$.

keys and other species (Van Brederode et al., 1990; Carder et al., 1996; DeFelipe, 1997). Double immunofluorescence for CaMKIV (red fluorophore) and parvalbumin (green fluorophore) confirmed that CaMKIV is clearly expressed in this subgroup of GABAergic neurons in all layers in which parvalbumin was present (Fig. 8C). However, we also observed that a number of neurons that were immunostained with either antiparvalbumin or anti-CaMKIV failed to clearly colocalize, suggesting that CaMKIV expression occurs in a mixed group of neurons. Interestingly, double immunofluorescence for CaMKIV (red fluorophore) and calbindin (green fluorophore) showed that CaMKIV is mostly excluded from this other subgroup of GABAergic neurons (Fig. 8D). Finally, double immunofluorescence for CaMKIV (red fluorophore) and calretinin (green fluorophore) revealed that CaMKIV is also colocalized to this subgroup of neurons (Fig. $8 E$ ). Together, these results strongly suggest that a large proportion of the dynamic nuclear CaMKIVimmunopositive signal seen in area $\mathrm{V} 1$ of adult monkeys after ME is localized to specific populations of smooth nonpyramidal neurons. However, because of the strong presence of the nuclear CaMKIV signal in the granular layers, it is most likely that other subgroups of cortical interneurons, perhaps glutaminergic excitatory interneurons such as spiny stellate cells, may also express nuclear concentration of CaMKIV after ME. This last point currently remains conjectural because of the absence of a reliable marker for this neuronal subgroup.

\section{Discussion}

\section{Interneuronal specificity of CaMKIV expression in area V1}

CaMKIV was first identified in rat cerebellum, where it is expressed in granule cells, an excitatory neuron subtype, as well as Purkinje cells, a major inhibitory subclass (Ohmstede et al., 1989;
Jensen et al., 1991b). However, the expression pattern of CaMKIV has, to date, not been reported for the primate primary visual cortex. In this study, we have presented several lines of evidence to show that CaMKIV expression in the monkey area $\mathrm{V} 1$ is primarily restricted to interneurons. First, CaMKIV was expressed in area V1 neurons that were morphologically distinct from the immunopositive cells observed in the pyramidal layer of the hippocampus CA1 region. We found CaMKIV immunostaining in monkey hippocampus to be similar to that in other species in two respects: the signal was confined to pyramidal neurons and various cellular compartments, including the cytoplasm, nuclei, and apical dendrites, showed an antigenic reaction (Jensen et al., 1991a; Kang et al., 2001). Second, we observed extensive nuclear CaMKIV staining in layer IVC of area V1, where pyramidal neurons are known to be almost completely absent (Mates and Lund, 1983). Third, colocalization experiments with three different markers of inhibitory neurons (anti-GABA, anti-parvalbumin, and anti-calretinin) clearly showed coincidental immunostaining with a large proportion of the nuclear CaMKIV signal in area V1. Colocalization of the nuclear CaMKIV signal with parvalbumin and calretinin is especially noteworthy because these markers have been linked previously to specific neuronal subtypes in the neocortex. Specifically, parvalbumin-expressing neurons are most commonly chandelier cells, whereas calretinin-expressing neurons are most frequently Cajal-Retzius cells (DeFelipe, 1997). Together, these results suggest that neurons with horizontal connectivity in area V1 of adult monkeys may be specifically influenced by prolonged periods of ME.

\section{Dynamic increase of nuclear staining of CaMKIV in area V1 after ME}

The basal expression of CaMKIV in normal adult V1 was mostly confined to the cytoplasm in all layers, except layer VI, in which marginal nuclear staining was observed. In response to $\mathrm{ME}$, we observed a notable increase in CaMKIV staining within the nucleus. Our results showed that nuclear CaMKIV expression changed in a laminar-specific and temporally specific manner. For example, after a short duration of $\mathrm{ME}$ ( $5 \mathrm{hr}, 1 \mathrm{~d})$, nuclear CaMKIV immunostaining increased exclusively within the extragranular layers (layers II/III and VI). However, a striking change in the laminar pattern of nuclear CaMKIV staining occurred after longer-term ME ( 5 and $30 \mathrm{~d}$ ). At this point, an increase in nuclear CaMKIV was observed within the granular layer subcompartments, notably layers IVC $\alpha$ and IVC $\beta$. CaMKIV expression seemed to be columnar only at the $30 \mathrm{~d}$ ME time point. CaMKIV columns were coincidental with Zif268-immunopositive columns, indicating a greater response linkage to the open-eye columns of area V1. Together, these results suggest that diminished inhibitory transmission from intermediate neurons between open-eye and closed-eye columns may cause the increase of the nuclear CaMKIV signal. However, the nuclear CaMKIV signal at longer time points (e.g., $30 \mathrm{~d} \mathrm{ME}$ ) in the open-eye columns sug- 

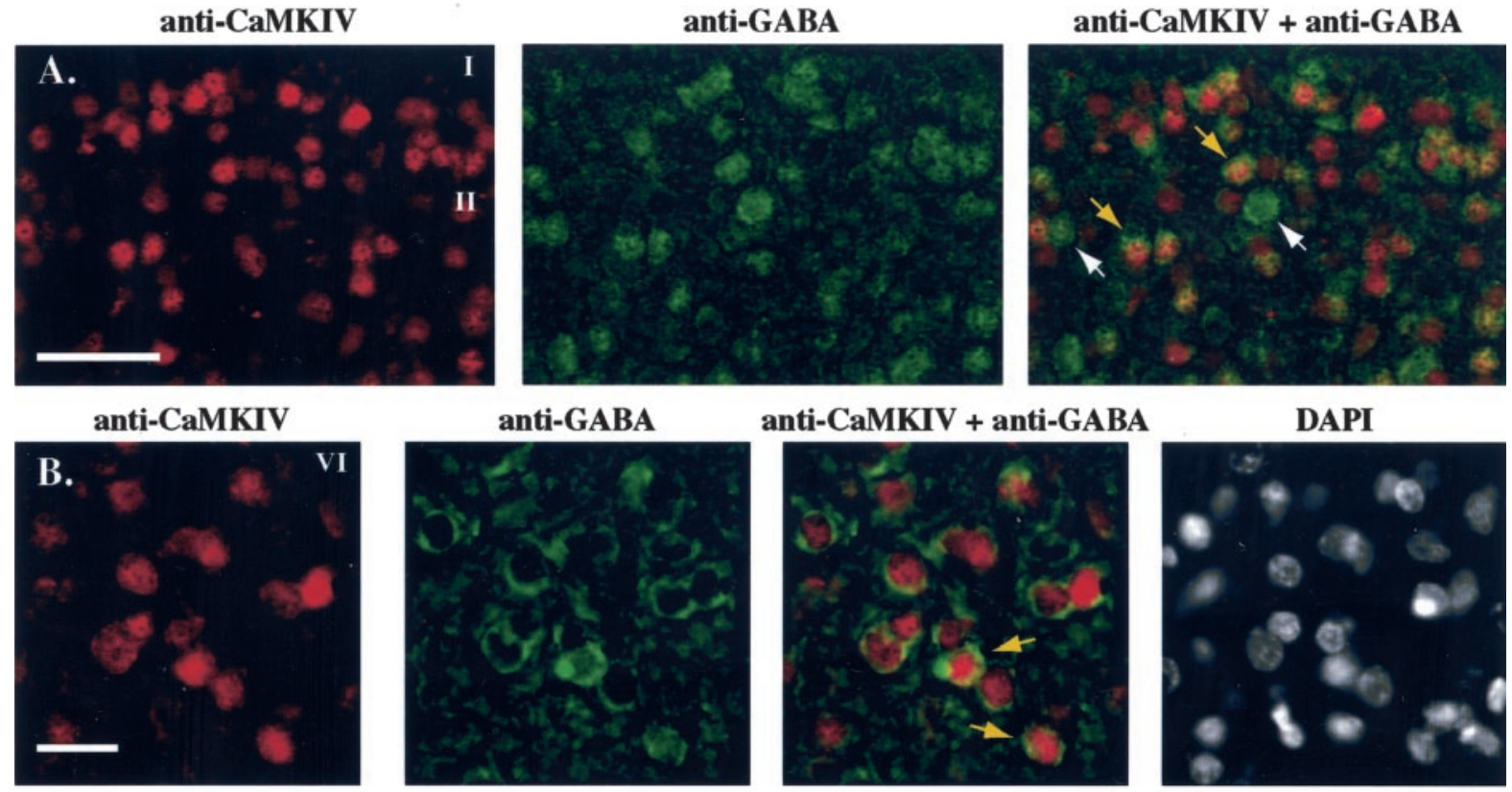

DAPI
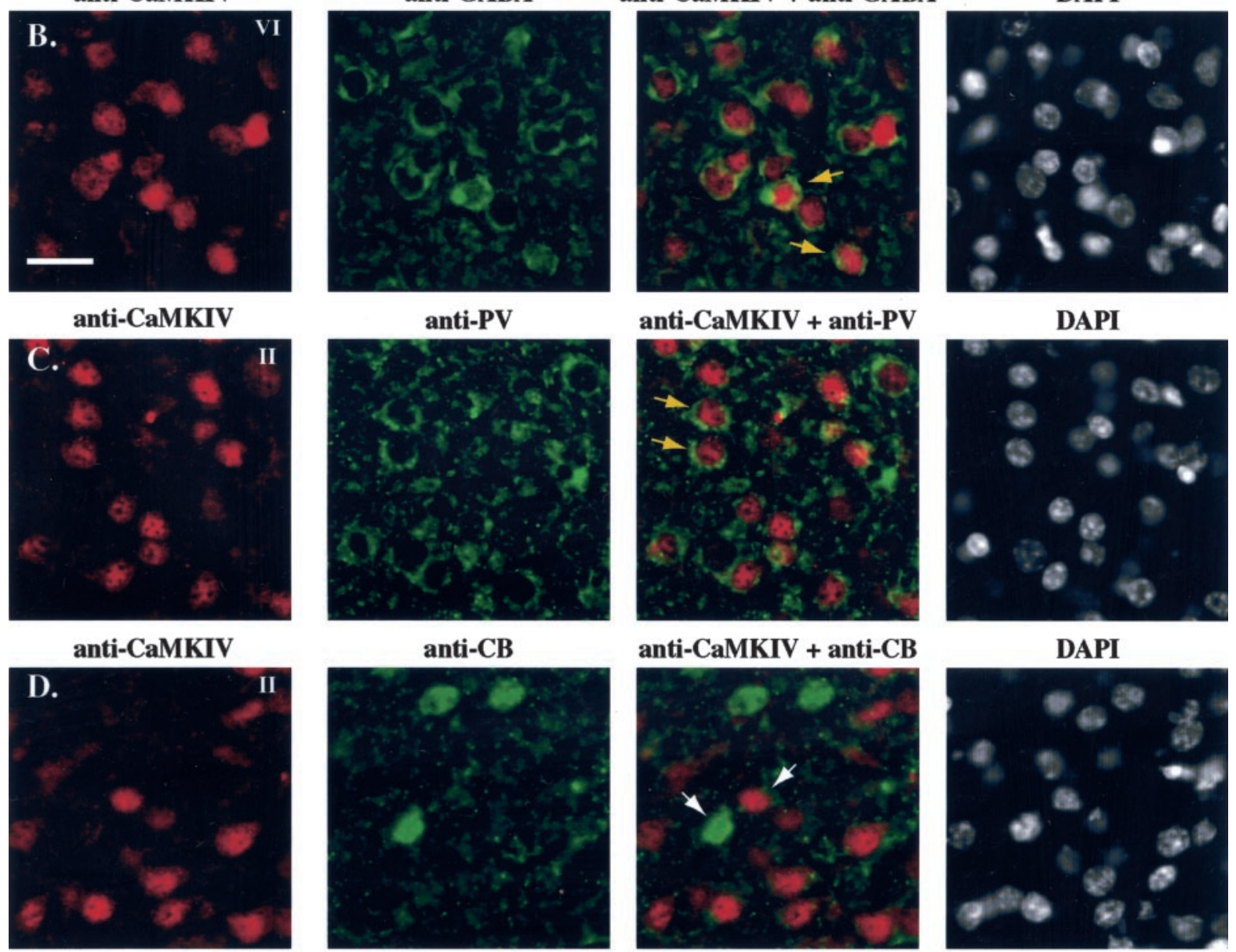

anti-CaMKIV

anti-CR
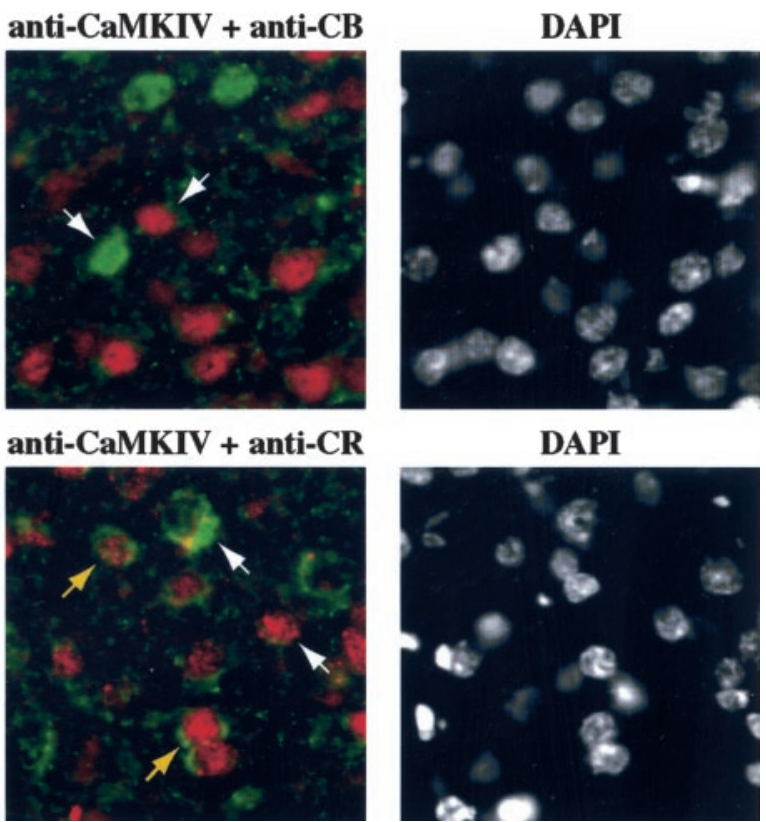

\section{DAPI}
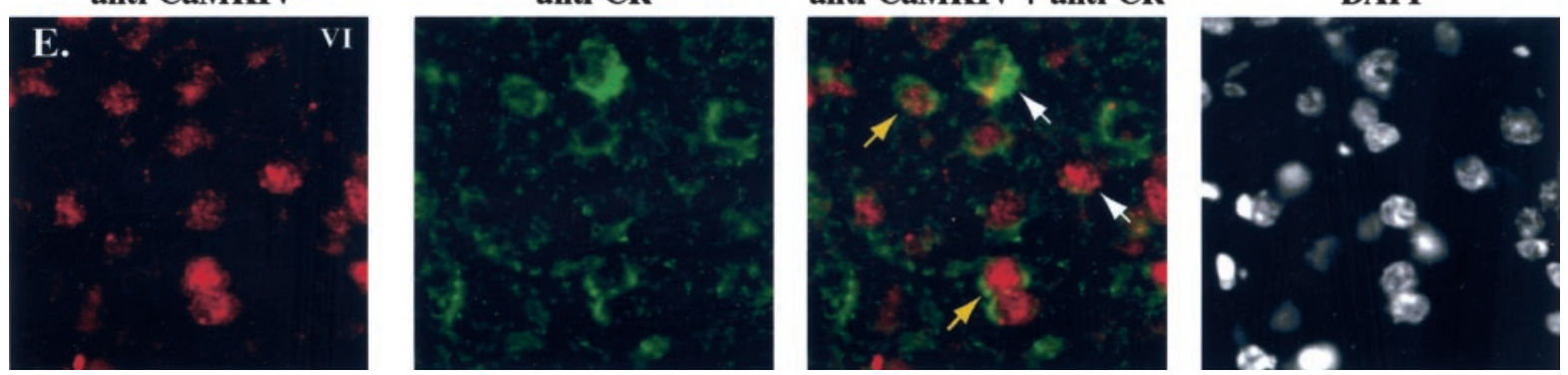

Figure 8. Nuclear CaMKIV signal colocalized with GABAergic neurons and other markers of interneurons in area V1 of ME animals. $A, B$, Examples of immunohistochemical staining of CaMKIV with two different GABA antisera (green). The CaMKIV nuclear signal was colocalized with numerous GABA-positive neurons in layer II of an adult $1 \mathrm{~d}$ ME monkey (A, rabbit anti-GABA). A similar colocalized signal was seen in layer VI of an adult $5 \mathrm{~d}$ ME monkey with a different antiserum ( $B$, guinea pig anti-GABA). Comparable patterns of colocalized staining were observed in other layers and ME time points (data not shown). A subset of CaMKIV nuclei (red) partially colocalized with parvalbumin ( $C$, green) but not calbindin ( $D$, green), two other known markers of GABAergic neurons. $E$, CaMKIV (red) also colocalized with a subpopulation of neurons immunopositive for calretinin (green). White arrows in all panels represent the absence of colocolization for any two signals in double-labeling experiments. Yellow arrows indicate double immunohistochemical nuclear staining for CaMKIV (red) and staining for GABA, parvalbumin, calbindin, or calretinin (green). Scale bars: $A, 50 \mu \mathrm{m} ; B-E, 25 \mu \mathrm{m}$. 
gests that the diminished influence of inhibitory transmission from the closed-eye columns may outlast the influence from the open-eye columns.

Our results on the laminar and temporal expression profiles of CaMKIV in the adult monkey are of particular interest because of the known dynamic neuroplastic properties of infant sensory cortex. Electrophysiological evidence suggests that neuroplastic changes in developing primary visual cortex in response to a brief period of monocular deprivation $(24 \mathrm{hr}$ ) are initiated in the extragranular layers before extending to the granular layer (Trachtenberg et al., 2000). Interestingly, we observed a similar dynamic change in CaMKIV nuclear localization after ME in adult monkeys. The laminar specificity of nuclear CaMKIV expression may, therefore, reflect a greater susceptibility of the extragranular layers to engage CaMKIV-guided molecular events that occur only in response to alterations in sensory input.

These findings contrast with the CaMKIV staining pattern observed in infant monkey area V1. The major difference to note is that CaMKIV expression showed significant nuclear compartmentalization in normal infant area V1. Thus, sensory input disruption by way of ME did not specifically produce any detectable changes in the infant laminar profile of nuclear CaMKIV as detected in the adult. It may be that similar laminar changes are also induced in the infant in response to ME but that they occur within the context of a constitutively elevated nuclear background of CaMKIV. A putative molecular role of CaMKIV in guiding neuroprotective and neuroplastic mechanisms would explain the high basal nuclear expression of CaMKIV in infants attributable to ongoing activity-driven maturation of the cortex. The dynamic changes evident in the adult may, therefore, initiate a comparable series of CaMKIV-guided molecular events, but only in response to selective sensory disruption and unfolding on a longer time scale ( $>1 \mathrm{~d}$ of $\mathrm{ME})$.

\section{Molecular functions of CaMKIV in neurons}

CaMKIV-mediated activity is known to play a role in neuroprotection (Bae et al., 2003; Sée et al., 2001) as well as in neuroplastic events associated with late phase LTD, LTP, and consolidation/ retention of memory (Ho et al., 2000; Kang et al., 2001; Wei et al., 2002). A clue as to how CaMKIV may be involved in these functions can be taken from its role in mediating calcium-induced dendritic growth in cortical neurons (Redmond et al., 2002). It was shown that either CaM kinase inhibitors or insertion of a kinase-dead form of CaMKIV suppresses dendritic growth despite calcium influx into neurons. However, a constitutively active form of CaMKIV induces dendritic growth in cultures via CREB-mediated signaling to the nucleus despite the absence of extracellular stimulation (Redmond et al., 2002).

The above studies provide strong evidence that CaMKIVguided molecular events can induce modification of cortical dendritic structures through a CREB-dependent mechanism. In vitro studies have shown that CaMKIV regulates several downstream molecules through phosphorylation, including most notably CREB at Ser-133 (Mathews et al., 1994; Sun et al., 1994) and CREB-binding protein (CBP) at Ser-301 (Chawla et al., 1998; Impey et al., 2002). CBP is a coactivator for CREB and increases the ability of phosphorylated CREB to activate transcription of cAMP-responsive genes (Roland et al., 1994). The trigger for CBP/CREB transactivation originates with calcium influx that in turn activates CaMKII, CaMKIV, and PKA (Hu et al., 1999). However, it is specifically CBP phosphorylation by CaMKIV that is necessary for long-term, persistent CREB-dependent gene regulation (Impey et al., 2002).
A role for CaMKIV in cortical neuroprotection and plasticity? It is likely that CaMKIV may play a similar neuroprotective or neuroplastic role in the neocortex. One possibility is that CaMKIV may be a central participant in normal, ongoing activity-driven plastic maturation of sensory neocortex. Such a role is consistent with our findings in infant area V1, where a high basal concentration of CaMKIV was observed within neuronal nuclei. The transcriptional targets of CaMKIV-mediated CBP/ CREB phosphorylation may be required to orchestrate the morphological and physiological events that are evident in developmental plasticity.

Our results in area V1 with regard to CaMKIV expression are especially tantalizing in the context of adult neocortical plasticity. There is now accumulating evidence that adult sensory cortex retains a certain degree of activity-induced plastic malleability that allows it to make adaptive changes in response to dynamically changing inputs (Katz and Shatz, 1996; Sugita, 1996; Zito and Svoboda, 2002). A reasonable postulate in this regard is that similar molecular mechanisms as in the developing neocortex may be recruited in the adult as well, although perhaps with different degrees of efficacy. Our observation of increasing nuclear concentration of CaMKIV in the adult after ME, in conjunction with its known molecular functions, suggests that CaMKIV may play a neuroprotective or neuroplastic role in the mature neocortex. There are four points of particular interest in this regard.

First, the nuclear localization of CaMKIV in adult area V1 increased after ME, whereas it was diffusely expressed throughout various neuronal compartments in normal animals. This suggests that a nuclear translocation may accompany $\mathrm{ME}$ and, therefore, induce CaMKIV downstream effects in an activity-driven manner. Second, not all neurons with a cytoplasmic CaMKIVpositive signal necessarily showed strong nuclear staining at all ME time points. In fact, even after 5 and $30 \mathrm{~d}$ of ME, a number of neurons continued to display only a cytoplasmic CaMKIV signal. Given that our colocalization experiments showed neurons with a strong nuclear CaMKIV signal to be homogeneous subpopulations of neurons, it may be that distinct neuronal subtypes of area V1 at different ME time points are particularly sensitive to disruption of activity. Third, our findings of columnar expression in long-term enucleated animals suggest that the activity-mediated functions of CaMKIV are engaged over a prolonged period. This result is consistent with a previous report that CaMKIV is necessary for long-term, persistent CREB-dependent gene regulation (Impey et al., 2002). Fourth, the laminar progression of nuclear CaMKIV expression in the adult after ME concurs with electrophysiological studies in infant visual cortex (Trachtenberg et al., 2000) and adult somatosensory cortex (Diamond et al., 1994) for an extragranular to granular layer progression of activitydependent neuroplastic changes.

In conclusion, our findings provide strong correlative evidence for a key molecular role of CaMKIV in neocortical plasticity. A putative role for CaMKIV in the infant brain in this regard is not surprising. However, it is our findings in the adult primary visual cortex that provide a potentially new perspective concerning the molecular coordination of neuroplastic reorganization. In this respect, we propose that CBP/CREB gene transcription driven by CaMKIV activity within distinctive groups of interneurons may play an important role in adaptive plastic reorganization of adult neocortical tissue. Although the precise functional impact of CaMKIV activity in the neocortex remains unknown, it is likely that its major roles may be similar to those seen in other brain areas [i.e., the preservation of neuronal integrity (neuro- 
protection) in conjunction with directing the molecular program for physiological and morphological changes (neuroplasticity) that accompany prolonged disruption of sensory input].

\section{References}

Ahn S, Ginty DD, Linden DJ (1999) A late phase of cerebellar long-term depression requires activation of CaMKIV and CREB. Neuron 23:559-568.

Bae JS, Jang MK, Hong SH, An WG, Choi YH, Kim HD, Cheong JH (2003) Phosphorylation of NF- $\kappa$ B by calmodulin-dependent kinase IV activates anti-apoptotic gene expression. Biochem Biophys Res Commun 305:1094-1098.

Carder RK, Leclerc SS, Hendry SH (1996) Regulation of calcium-binding protein immunoreactivity in GABA neurons of macaque primary visual cortex. Cereb Cortex 6:271-287.

Celio MR, Baier W, Scharer L, de Viragh PA, Gerday C (1988) Monoclonal antibodies directed against the calcium binding protein parvalbumin. Cell Calcium 9:81-86.

Celio MR, Baier W, Scharer L, Gregersen HJ, de Viragh PA, Norman AW (1990) Monoclonal antibodies directed against the calcium binding protein calbindin D-28k. Cell Calcium 11:599-602.

Chaudhuri A, Zangenehpour S (2002) Molecular activity maps of sensory function. In: Immediate early genes and inducible transcription factors in mapping of the central nervous system function and dysfunction (Kaczmarek L, Robertson HA, eds), pp 103-145. Boston: Elsevier Science.

Chaudhuri A, Matsubara JA, Cynader MS (1995) Neuronal activity in primate visual cortex assessed by immunostaining for the transcription factor Zif268. Vis Neurosci 12:25-50.

Chawla S, Hardingham GE, Quinn DR, Bading H (1998) CBP: a signal transcriptional coactivator controlled by nuclear calcium and CaM kinase IV. Science 281:1505-1509.

DeFelipe J (1997) Type of neurons, synaptic connections and chemical characteristics of cells immunoreactive for calbindin-D28K, parvalbumin and calretinin in the neocortex. J Chem Neuroanat 14:1-19.

Diamond ME, Huang W, Ebner FF (1994) Laminar comparison of somatosensory cortical plasticity. Science 265:1885-1888.

Frangakis MV, Chatila T, Wood ER, Sahyoun N (1991) Expression of a neuronal $\mathrm{Ca}^{2+} /$ calmodulin-dependent protein kinase, CaM kinase-Gr, in rat thymus. J Biol Chem 266:17592-17596.

Herdegen T, Kovary K, Leah JD, Bravo R (1991) Specific temporal and spatial distribution of JUN, FOS, and KROX-24 proteins in spinal neurons following noxious transsynaptic stimulation. J Comp Neurol 313:178-191

Ho N, Liauw JA, Blaeser F, Wei F, Hanissian S, Muglia LM, Wozniak DF, Nardi A, Arvin KL, Holtzman DM, Linden DJ, Zhuo M, Muglia LJ, Chatila TA (2000) Impaired synaptic plasticity and cAMP response element-binding protein activation in $\mathrm{Ca}^{2+} /$ calmodulin-dependent protein kinase type IV/Gr-deficient mice. J Neurosci 20:6459-6472.

Hof PR, Ungerleider LG, Webster MJ, Gattass R, Adams MM, Sailstad CA, Morrison JH (1996) Neurofilament protein is differentially distributed in subpopulations of corticocortical projection neurons in the macaque monkey visual pathways. J Comp Neurol 376:112-127.

Hu SC, Chrivia J, Ghosh A (1999) Regulation of CBP-mediated transcription by neuronal calcium signaling. Neuron 22:799-808.

Impey S, Fong AL, Wang Y, Cardinaux JR, Fass DM, Obrietan K, Wayman GA, Storm DR, Soderling TR, Goodman RH (2002) Phosphorylation of CBP mediates transcriptional activation by neural activity and CaM kinase IV. Neuron 34:235-244.

Jensen KF, Ohmstede CA, Fisher RS, Olin JK, Sahyoun N (1991a) Acquisition and loss of a neuronal $\mathrm{Ca}^{2+} /$ calmodulin-dependent protein kinase during neuronal differentiation. Proc Natl Acad Sci USA 88:4050-4053.

Jensen KF, Ohmstede CA, Fisher RS, Sahyoun N (1991b) Nuclear and ax- onal localization of $\mathrm{Ca}^{2+} /$ calmodulin-dependent protein kinase type $\mathrm{Gr}$ in rat cerebellar cortex. Proc Natl Acad Sci USA 88:2850-2853.

Jones DA, Glod J, Wilson-Shaw D, Hahn WE, Sikela JM (1991) cDNA sequence and differential expression of the mouse $\mathrm{Ca}^{2+} /$ calmodulindependent protein kinase IV gene. FEBS Lett 289:105-109.

Kang H, Sun LD, Atkins CM, Soderling TR, Wilson MA, Tonegawa S (2001) An important role of neural activity-dependent CaMKIV signaling in the consolidation of long-term memory. Cell 106:771-783.

Katz LC, Crowley JC (2002) Development of cortical circuits: lessons from ocular dominance columns. Nat Rev Neurosci 3:34-42.

Katz LC, Shatz CJ (1996) Synaptic activity and the construction of cortical circuits. Science 274:1133-1138.

Kogan CS, Zangenehpour S, Chaudhuri A (2000) Developmental profiles of SMI-32 immunoreactivity in monkey striate cortex. Dev Brain Res 119:85-95.

Lee PD, Sladek R, Greenwood CM, Hudson TJ (2002) Control genes and variability: absence of ubiquitous references transcripts in diverse mammalian expression studies. Genome Res 12:292-297.

Mates SL, Lund JS (1983) Neuronal composition and development in lamina 4C of monkey striate cortex. J Comp Neurol 221:60-90.

Mathews RP, Guthrie CR, Wailes LM, Zhao X, Means AR, McKnight GS (1994) Calcium/calmodulin-dependent protein kinase types II and IV differentially regulate CREB-dependent gene expression. Mol Cell Biol 14:6107-6116.

Ohmstede CA, Jensen KF, Sahyoun NE (1989) $\mathrm{Ca}^{2+} /$ calmodulindependent protein kinase enriched in cerebellar granule cells. Identification of a novel neuronal calmodulin-dependent protein kinase. J Biol Chem 264:5866-5875.

Redmond L, Kashani AH, Ghosh A (2002) Calcium regulation of dendritic growth via CaM kinase IV and CREB-mediated transcription. Neuron 34:999-1010.

Roland PS, Kwok JR, Lundblad JC, Richards JP, Bächinger HP, Brennan RG, Roberts SG, Green MR, Goodman RH (1994) Nuclear protein CBP is a coactivator for the transcription factor CREB. Nature 370:223-226.

Sée V, Boutillier AL, Bito H, Loeffler JP (2001) Calcium/calmodulindependent protein kinase type IV (CaMKIV) inhibits apoptosis induced by potassium deprivation in cerebellar granule neurons. FASEB J 15:134-144.

Seguela P, Geffard M, Buijs RM, Le Moal M (1984) Antibodies against gamma-aminobutyric acid: Specificity studies and immunocytochemical results. Proc Natl Acad Sci USA 81:3888-3892.

Soderling TR (2000) CaM-kinases: modulators of synaptic plasticity. Curr Opin Neurobiol 10:375-380.

Sternberger LA, Sternberger NH (1983) Monoclonal antibodies distinguish phosphorylated and nonphosphorylated forms of neurofilaments in situ. Proc Natl Acad Sci USA 80:6126-6130.

Sugita Y (1996) Global plasticity in adult visual cortex following reversal of visual input. Nature 380:523-526.

Sun P, Enslen H, Myung PS, Maurer RA (1994) Differential activation of CREB by $\mathrm{Ca}^{2+} /$ calmodulin-dependent protein kinases type II and type IV involves phosphorylation of a site that negatively regulates activity. Genes Dev 8:2527-2539.

Trachtenberg JT, Trepel C, Stryker MP (2000) Rapid extragranular plasticity in the absence of thalamocortical plasticity in the developing primary visual cortex. Science 287:2029-2032.

Van Brederode JF, Mulligan KA, Hendrickson AE (1990) Calcium-binding proteins as markers for subpopulations of GABAergic neurons in monkey striate cortex. J Comp Neurol 298:1-22.

Wei F, Qiu CS, Liauw J, Robinson DA, Ho N, Chatila T, Zhuo M (2002) Calcium-calmodulin-dependent protein kinase IV is required for fear memory. Nat Neurosci 5:573-579.

Zito K, Svoboda K (2002) Activity-dependent synaptogenesis in the adult mammalian cortex. Neuron 35:1015-1017. 